\title{
The capability of B-RISK zone modelling software to simulate BRE multiple vehicle fire spread test
}

\begin{abstract}
Building Research Establishment (BRE), United Kingdom have carried out several full-scale experiments of vehicle fire as to address the fire spread between vehicles. Thus, this paper aims to investigate the capability of the B-RISK zone modelling software to simulate the BRE multiple vehicle fire spread test. Using the information gathered from the work by BRE, series of simulations have been conducted. The results of the simulations are compared with the results from the experiments. Analysis shows that the predicted results from the B-RISK simulations give slightly faster time of ignition to the ones obtained using hand calculation. This could be due to B-RISK includes the radiation effect from the underside of the hot upper layer. As a conclusion, the analysis shows that using the B-RISK simulation software with additional radiation effects does not improve the result as compared to using the hand calculation considering the level of uncertainties which required to be assumed on some input parameters e.g. HRRPUA, heat of combustion, and/or latent heat of gasification.
\end{abstract}

Keyword: Zone model; Vehicle fire; Fire spread simulation 